\title{
ANTIMICROBIAL SUSCEPTIBILITY PATTERNS OF PSEUDOMONAS SPECIES ISOLATED FROM VARIOUS CLINICAL SAMPLES AT A TERTIARY CARE HOSPITAL
}

\author{
Rabin Gyawali ${ }^{1}$, Ram Bahadur Khadka ${ }^{2}$, Basudha Shrestha ${ }^{3}$, Sarita Manandhar ${ }^{4 *}$ \\ ${ }^{I}$ National College, Tribhuvan University (affiliated), Khusibu, Kathmandu, Nepal \\ ${ }^{2}$ Department of Health Science, Crimson College of Technology, Pokhara University, Nepal \\ ${ }^{3}$ Department of Microbiology, Kathmandu Model Hospital, Kathmandu, Nepal \\ ${ }^{4}$ Department of Microbiology, Tri-Chandra Multiple Campus, Tribhuvan University, Nepal \\ "Corresponding author: sarita111@gmail.com
}

(Received: June 13, 2020; Revised: August 30, 2020; Accepted: October 05, 2020)

\begin{abstract}
Considerable increase in the prevalence and multidrug-resistant (MDR) Pseudomonas has been observed with towering morbidity and mortality. As a consequence of the haphazard use of antimicrobials, the spread of antimicrobial resistance is now a global issue. This study aimed to access the distribution rate and antibiotic susceptibility patterns of Pseudomonas species isolated from various clinical specimens in Kathmandu Model Hospital, Nepal. During the study period, 1252 samples were collected, cultured and the organism was isolated and identified. The antimicrobial susceptibility testing was done using the modified Kirby-Bauer disc diffusion method as per CLSI guidelines. Out of 1252 samples, 28 clinical isolates of Pseudomonas species were isolated. The highest number of Pseudomonas spp. was isolated from swab samples that included pus, ear, and wound (46.4\%). Pseudomonas spp. demonstrated marked resistance against cefixime $(96.4 \%)$ and showed higher sensitivity to piperacillin/tazobactam $(92.9 \%)$. The result showed pus, wound exudates, ear discharges samples exhibit Pseudomonas as common etiology of infection. Pseudomonas spp. demonstrated highest sensitivity against piperacillin/tazobactam, amikacin, meropenem, gentamycin. The steady resistance of Pseudomonas spp. to most of the antibiotics, necessitates these drugs to be confined to extreme infections and hospital intensive care units to circumvent the speedy emergence of resistant strains.
\end{abstract}

Keywords: Antibiotic resistance, Cefixime, Kirby-Bauer disc diffusion, Multi drug resistance, Pseudomonas.

\section{INTRODUCTION}

Pseudomonas species are Gram-negative, aerobic, nonspore-forming straight or slightly rod-shaped bacteria that belong to the family Pseudomonadacae. It is extensively prevalent in nature including soil, aqua, and diverse flora all around the globe (Kireçci \& Kareem, 2014; Pathmanathan et al., 2009). The most important species from a medical point of view is Pseudomonas aeruginosa $(P$. aeruginosa) while Pseudomonas fluorescens $(P$. fluorescens) and Pseudomonas putida ( $P$. putida) occasionally cause infection in immunosuppressed hosts (Sivanmaliappan \& Sevanan, 2011; Kayser et al., 2005). Pseudomonas infections can flourish in multitudinous anatomical sites including skin, subcutaneous tissue, bones ears, eyes, urinary tract, respiratory tract, cardiac valves. The site may differ with the portal of entry and the patients' susceptibility (Kireçci \& Kareem, 2014).

Pseudomonas infections are often life-threatening and tough to manage due to intrinsic resistance to numerous antimicrobials. Systems responsible for antimicrobial drug resistance in Pseudomonas spp. are the resistance genes they harbor via. horizontal gene transfer, mutation in target sites and efflux proteins (Anil \& Shahid, 2013). Pseudomonas spp. exhibits resistance to numerous antibiotics, thereby jeopardizing the selection of convenient therapy (Javiya et al., 2008). Since novel antimicrobials agents may not be promptly available in near future, it is necessary to perform region-specific survey studies to generate data that would aid clinicians to determine the accurate empirical regimen (Javiya et al., 2008; Ramana \& Chaudhury, 2012).

In a country like Nepal, the haphazard use of antimicrobials plays an important role in the development of resistant nosocomial pathogens like Pseudomonas. Data on the antimicrobial susceptibility profile of Pseudomonas spp. is not ample in Nepal. Continued studies on antimicrobial resistance profile of Pseudomonas are crucial to determine susceptible pattern against regularly prescribed antimicrobials in Nepal and guide the clinicians to select the treatment options (Anil \& Shahid, 2013). Therefore, this cross-sectional study determines the distribution rate and antimicrobial susceptibility patterns of Pseudomonas spp. isolated from various clinical samples of patients in Kathmandu Model Hospital.

\section{MATERIALS AND METHODS}

Study design, specimen collection, and bacterial identification

A descriptive cross-sectional study was undertaken at the Department of Microbiology in Kathmandu Model Hospital to isolate the bacteria and verify their antibiotic 
susceptibility pattern. Twelve hundred and fifty-two samples which include urine, sputum, swab (pus, wound and ear), fluid, tips (catheter, VP shunt) were collected from patients of all ages and both genders excluding the patients already on antibiotic therapy. Properly labeled samples were obtained in a clean, sterile, and leak proof container. The samples were inoculated on MacConkey Agar and Blood Agar, and the bacteria were identified by colony morphology, Gram staining, biochemical tests; which included indole test, methyl red test, VogesProskauer test, citrate test, triple sugar iron test, oxidase test, and green-blue pigment production test (Golshani et al., 2012).

\section{Antibiotic susceptibility testing}

Antibiotic susceptibility testing was performed by modified Kirby Bauer's disc diffusion method on Mueller Hinton Agar medium according to the Clinical Laboratory Standard Institute guidelines (CLSI, 2013). The antibiotics used were ciprofloxacin $((5 \mu \mathrm{g})$, cotrimoxazole $(25 \mu \mathrm{g})$, cefotaxime $(30 \mu \mathrm{g})$, ceftriaxone $(30 \mu \mathrm{g})$, cefixime (10 $\mu \mathrm{g})$, amikacin $(30 \mu \mathrm{g})$, gentamycin $(10 \mu \mathrm{g})$, ceftazidime $(30 \mu \mathrm{g})$, cefoperazone/sulbactum $(75 / 10 \mu \mathrm{g})$, meropenem $(10 \mu \mathrm{g})$, piperacillin/tazobactam $(100 / 10 \mu \mathrm{g})$, chloramphenicol $(30 \mu \mathrm{g})$ (HiMedia, India). Suspension of bacteria maintained to $0.5 \mathrm{McFarland}$ standards was inoculated on Mueller Hinton Agar (HiMedia, India) plates using sterile swabs, and then antibiotic discs were placed on it. The plates were incubated at $37{ }^{\circ} \mathrm{C}$ for 24 hours. The diameter of the zone of inhibition was measured and compared with standard strain. The results were interpreted as sensitive, intermediate, resistant according to CLSI (2013) guidelines. Pseudomonas (ATCC 27853) was used as standard control strains.

\section{Data analysis}

All the results were entered in the worksheet of Statistical Package for Social Science Software (SPSS 14.0) and compared by the chi-square test as percentages with $95 \%$ confidence intervals. A $p$ value of $<0.05$ was considered to be statistically significant.

\section{RESULTS}

Among 1252 clinical samples, a total of 28 (2.2\%) Pseudomonas spp. was isolated. With regards to gender, $16(57.1 \%)$ isolates were from male patient while 12 $(42.9 \%)$ from female patient (Fig. 1). Isolation of Pseudomonas spp. with gender of patient was not statistically significant. The highest number of isolates $(\mathrm{n}=13,46.4 \%)$ were from the age group of 16-30 years and the least $(n=2,7.1 \%)$ were from 46-60 years of age. Five $(17.9 \%)$ isolates were from age group of $31-45$ years, $4(14.3 \%)$ from less than 15 years and above 60 years of age (Fig. 2). The percentage of isolation of Pseudomonas spp. with age of patient was not statistically significant.

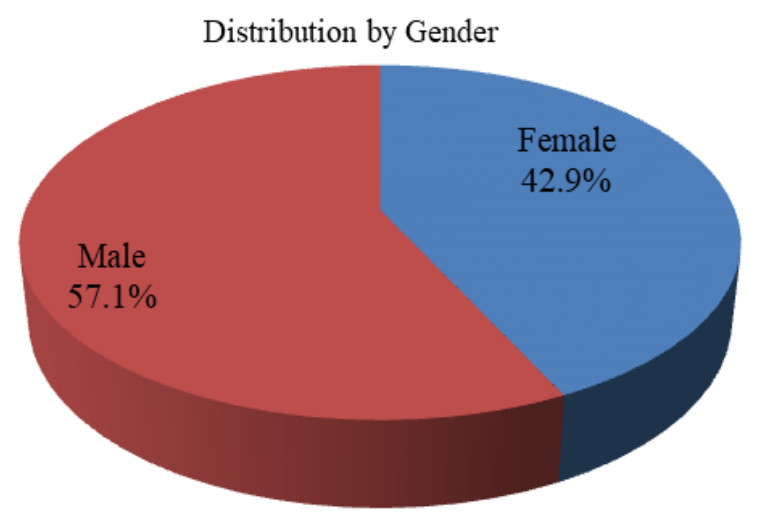

Fig. 1. Demographic characteristics of patients

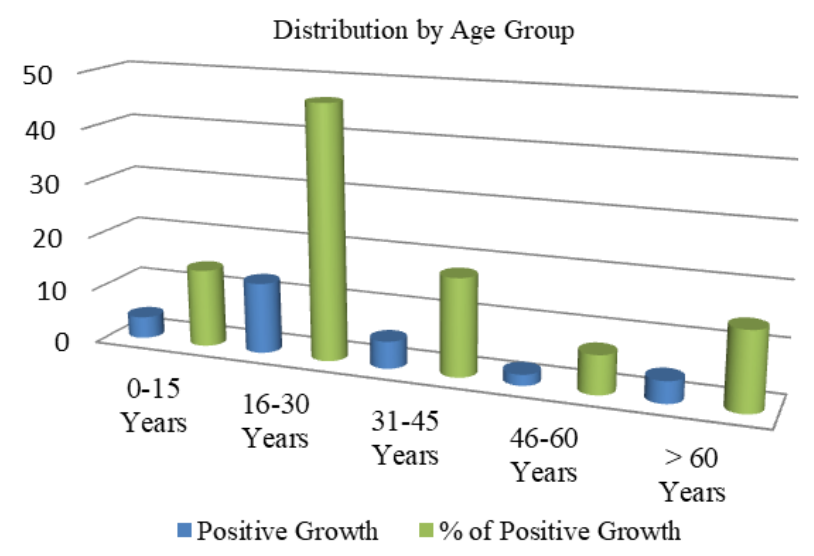

Fig. 2. Age-wise distribution of patients

Out of 1083 samples collected from outpatients, a total number of isolates were $18(1.66 \%)$, and out of 169 samples collected from inpatients, the total number of isolates was $10 \quad(5.91 \%)$ and the distribution of Pseudomonas spp. with patient settings was statistically significant (Table 1). Thirteen (46.4 \%) isolates of Pseudomonas spp. were contributed from swab sample, 8 $(28.6 \%)$ from urine sample, $4(14.3 \%)$ from sputum sample, $2(7.1 \%)$ from tips that include catheter tip and VP shunt tip, and 1 (3.6 \%) from the fluid sample. The isolated of Pseudomonas spp. with different samples was statistically significant (Table 2).

Pseudomonas spp. were susceptible to piperacillin/tazobactam $26(92.9 \%)$ followed by amikacin $24(85.7 \%)$, meropenem and gentamycin $19(67.9 \%)$, ciprofloxacin $18(64.3 \%)$, ceftazidime $16(57.1 \%)$, cefotaxime $14(50.0 \%)$, ceftriaxone $7(25 \%)$ and cotrimoxazole $4(14.3 \%)$. Pseudomonas isolates were resistant to cefixime $27(96.4 \%)$ followed by cotrimoxazole $21 \quad(75 \%)$, chloramphenicol and cefuperazone/sulbactam $20(71.46 \%)$, ceftriaxone 16 $(57.1 \%)$, cefotaxime $9(32.1 \%)$, gentamycin $8(28.6 \%)$, ciprofloxacin and ceftazidime $7(25 \%)$ and amikacin 3 (10.7\%), as depicted in Table 3. 
Table 1. Distribution of Pseudomonas spp. in outpatient and inpatient

\begin{tabular}{lcccc}
\hline \multirow{2}{*}{ Patient } & \multirow{2}{*}{ No. of Cases } & Negative (n, \%) & Positive (n, \%) & p-value \\
\cline { 3 - 5 } & 1083 & $1065,98.33$ & $18,1.66$ & \\
\hline Outpatient & 169 & $159,94.08$ & $10,5.91$ & 0.001 \\
Inpatient & 1252 & 1224 & $28(2.23 \%)$ & \\
Total & &
\end{tabular}

Table 2. Distribution of Pseudomonas spp. in different samples

\begin{tabular}{|c|c|c|c|c|c|}
\hline \multirow[b]{2}{*}{ Samples } & \multirow[b]{2}{*}{ No. of Cases } & \multicolumn{3}{|c|}{ Positive Growth } & \multirow[b]{2}{*}{$p$-value } \\
\hline & & No. & $\%$ & $\%$ of total & \\
\hline Urine & 870 & 8 & 28.6 & 0.63 & \multirow{6}{*}{0.002} \\
\hline Sputum & 92 & 4 & 14.3 & 0.31 & \\
\hline Fluid & 92 & 1 & 3.6 & 0.07 & \\
\hline Swab & 156 & 13 & 46.4 & 1.03 & \\
\hline Tips & 42 & 2 & 7.1 & 0.15 & \\
\hline Total & 1252 & 28 & 100 & 2.23 & \\
\hline
\end{tabular}

Table 3. Antimicrobial susceptibility pattern of Pseudomonas spp. isolates against first-line and second-line drugs

\begin{tabular}{|c|c|c|c|c|c|c|c|}
\hline \multirow[b]{3}{*}{ Class of Antibiotics } & \multirow{3}{*}{ Potency $(\mu \mathrm{g})$} & \multicolumn{6}{|c|}{ Susceptibility Patterns of Pseudomonas spp. $(\mathrm{N}=28)$} \\
\hline & & \multicolumn{2}{|c|}{ Sensitive } & \multicolumn{2}{|c|}{ Intermediate } & \multicolumn{2}{|c|}{ Resistant } \\
\hline & & Total No. & $\%$ & Total No. & $\%$ & Total No. & $\%$ \\
\hline Fluoroquinolones & 5 & 18 & 64.3 & 3 & 10.7 & 7 & 25 \\
\hline \multicolumn{8}{|l|}{ Ciprofloxacin } \\
\hline Sulfonamides & 25 & 4 & 14.3 & 3 & 10.7 & 21 & 75 \\
\hline \multicolumn{8}{|l|}{ Cotrimoxazole } \\
\hline Aminoglycosides & 10 & 19 & 67.9 & 1 & 3.6 & 8 & 28.6 \\
\hline \multicolumn{8}{|l|}{ Gentamycin } \\
\hline Amikacin & 30 & 24 & 85.7 & 1 & 3.6 & 3 & 10.7 \\
\hline Cephalosporins & 30 & 16 & 57.1 & 5 & 17.9 & 7 & 25 \\
\hline \multicolumn{8}{|l|}{ Ceftazidime } \\
\hline Ceftriaxone & 30 & 7 & 25 & 5 & 17.9 & 16 & 57.1 \\
\hline Cefixime & 10 & 0 & 0 & 1 & 3.6 & 27 & 96.4 \\
\hline Cefotaxime & 30 & 14 & 50.0 & 5 & 17.9 & 9 & 32.1 \\
\hline Carbapenem & 10 & 19 & 67.9 & 0 & 0 & 9 & 32.1 \\
\hline \multicolumn{8}{|l|}{ Meropenem } \\
\hline Combination Drugs & $75 / 10$ & 6 & 21.4 & 2 & 7.1 & 20 & 71.4 \\
\hline \multicolumn{8}{|l|}{ Cephoperazone-Sulbactam } \\
\hline Piperacillin-Tazobactam & $100 / 10$ & 26 & 92.9 & 0 & 0 & 2 & 7.1 \\
\hline Miscellaneous & 30 & 8 & 28.6 & 0 & 0 & 20 & 71.4 \\
\hline Chloramphenicol & & & & & & & \\
\hline
\end{tabular}




\section{DISCUSSION}

During the study period, the highest number of Pseudomonas spp. was isolated from swab samples (46.4 $\%)$ followed by a urine sample $(28.6 \%)$ and sputum sample $(14.3 \%)$. The isolation of Pseudomonas spp. with different samples was statistically significant $(\mathrm{p}<0.05)$. The results are in line with other findings as Khan et al. (2008) showed maximum clinical isolates from pus swab $(57.64 \%)$ followed by urine sample $(24.2 \%)$ and Mohanasoundaram (2011) showed higher prevalence in pus swab followed by urine sample. The result was not consistent with the study carried out by Aggarwal et al. (2008) that showed the major source of Pseudomonas spp. $(28.57 \%)$ to be sputum and tracheostomy sample followed by pus $(24.13 \%)$, urine $(19.04 \%)$, and fluids $(15.38 \%)$. Wound infection, ear infection occurs on external body surfaces that make them vulnerable to contamination with dust, dirt, polluted water making the patient susceptible to community-acquired as well as hospital-acquired Pseudomonas spp. infection. Other parameters like poor health status, illiteracy, malnutrition, underlying diseases, and lack of proper techniques in using medical procedures may promote to Pseudomonas spp. infection in Nepal (Sharma et al., 2004).

The highest number of Pseudomonas isolates (46.4\%) was from the patients of the age group 16-30 years followed by $31-45$ years $(17.9 \%)$. There was no significant association of Pseudomonas spp. infection with age of patient $(\mathrm{P}>0.05)$. Findings of previous studies (Mohanasoundaram, 2011; Anil \& Shahid, 2013) reported the highest numbers of Pseudomonas isolates in different age groups. It could be due to the involvement of this age group in different practices, prolonged hospitalization, or decreased immunity (Anil \& Shahid, 2013). In this study, distribution of Pseudomonas isolates was common in males compared to females with a male to female ratio being 1.5:1. However, there was no significant association of Pseudomonas spp. infection with gender ( $\mathrm{P}>0.05)$. This result is in agreement with a previous study (Khan et al., 2008), which showed $61.78 \%$ male and $38.22 \%$ female infected by Pseudomonas. Many studies suggested that the Pseudomonas species infection is more common in male than female (Ullah et al., 2019).

Nowadays, the prevalence of Pseudomonas and the new resistant strains continue in both community and hospital originated infections (Savas et al., 2005). In this study, the highest number of cases was in inpatients as compared to outpatients. There was a significant association between outpatient/ inpatient and Pseudomonas spp. infection ( $\mathrm{p}<$ 0.05). Findings of previous studies conducted by Mohanasoundaram (2011) agree with the present study as they reported the highest number of cases in inpatients with $35.86 \%, 57 \%$, and $40.7 \%$ in the year 2008, 2009, and 2010 respectively. Pseudomonas spp. finds the hospital environment accommodating and is responsible for causing hospital-acquired infections. Also, inpatients are at risk due to underlying diseases, decreased immunity, and prolonged hospital stay. The use of equipment that requires a wet, body temperature environment, such as dialysis tubing and respiratory therapy equipment is particularly susceptible to colonization by the organism (Savas et al., 2005).

The unique feature of Pseudomonas spp. is its resistance to a variety of antibiotics that result from low permeability of the cell wall, the production of inducible cephalosporinases, an active efflux, and a poor affinity for the target DNA gyrase (Mohanasoundaram, 2011). In this study, Pseudomonas spp. isolates were more sensitive to aminoglycosides amikacin and gentamycin. These findings are in agreement with the findings of Savas et al. (2005); they reported that for Pseudomonas spp. the resistance rate against amikacin was very low, i.e., $26 \%$. These findings are similar to the findings of Kumar et al. (2020) and Tripathi et al. (2011) that showed less (26.66 $\%$ and $10.79 \%$ ) resistant rate to amikacin, respectively. Other studies reported that the antipseudomonal effects of amikacin were greater than gentamycin (Maes \& Vanhoof, 1992).

These findings are also similar to the findings of Chand et al. (2020) indicating higher sensitivity of gentamycin with a resistant rate of only $20.68 \%$. The result is not consistent with the findings of Agbo et al. (2020) and Motbainor et al. (2020) since they reported that for Pseudomonas the resistance rate against gentamycin was high i.e. $73.43 \%$ and $54.5 \%$, respectively. It might be due to the poor substrate property of these drugs for those enzymes that are responsible for adenylation or acetylation and phosphorylation. In this study, only $25 \%$ of Pseudomonas spp. showed resistance to ciprofloxacin that is comparable to studies of Anil and Shahid (2013), and Ramana and Chaudhury (2012) that showed $27.59 \%$ and $39 \%$ resistant to ciprofloxacin, respectively. The result is also comparable to recent studies of Agbo et al. (2020), Chand et al. (2020) and Kumar et al. (2020) which showed $21.68 \%, 31.03 \%$, and $35.55 \%$ resistant to ciprofloxacin, respectively, suggesting it could be the regular regimen for Pseudomonas spp infection.

Recently, increased resistance has been observed against third-generation cephalosporins for Gram-negative bacilli, especially $P$. aeruginosa. The present study also showed a high rate of resistance of isolates to cefixime $(96.4 \%)$ and ceftriaxone $(57.1 \%)$. These high values of resistance are comparable to the reports of Chand et al. (2020) and Motbainor et al. (2020) that showed $100 \%$ resistance to both cefixime and ceftriaxone. These findings are also similar to the studies of Anil and Shahid (2013) that revealed a higher resistance rate $(68.96 \%)$ to ceftriaxone. The increased prevalence of cephalosporin resistance is 
related to the increased use of beta-lactam antibiotics such as amoxicillin, enzymatic inactivation, and biofilm formation.

The present study also showed a higher resistance rate to co-trimoxazole $(75 \%)$. Less resistance to co-trimoxazole $(51.72 \%)$ had been reported in a study from Nepal (Anil $\&$ Shahid, 2013). High resistance to co-trimoxazole (100 $\%$ and $64.44 \%$ ) was reported in the studies of Motbainor et al. (2020) and Kumar et al. (2020), respectively. It might be due to alteration of the metabolic pathway by pathogen in which they might have bypassed the reactions of folic acid synthesis where the drug acts. Most Pseudomonas isolates were sensitive to piperacillin/tazobactam $(92.9 \%)$ followed by meropenem (67.9\%). Few Pseudomonas isolates were resistant to piperacillin/tazobactam $(7.1 \%)$ followed by meropenem $(32.1 \%)$. These results are similar to the study done by Tripathi et al. (2011) that showed $89.22 \%$ sensitive and $4.9 \%$ resistant isolates against piperacillin/tazobactam and $65.68 \%$ sensitive and $20.59 \%$ resistant against meropenem. Low rate of resistance to piperacillin/tazobactam (11.1\% and $19.4 \%)$ was reported by Kumar et al. (2020) and Chand et al. (2020), respectively. Similarly, low rate of resistance to meropenem was reported by Agbo et al. (2020). These drugs are needed to be preserved as antipseudomonal agents for future aspects.

In this study, Pseudomonas isolates were highly resistant to chloramphenicol and cefoperazone/ sulbactam $(71.4 \%)$ which was in corroboration with a report from India (Javiya et al., 2008) that showed a $75 \%$ resistance rate of Pseudomonas spp. to chloramphenicol. These results are not consistent with the study of Kumar et al. (2020) that showed only $20 \%$ resistance to cefoperazone/ sulbactam. Some data showed the antimicrobial resistances of $P$. aeruginosa to cefoperazone/ sulbactam ranged between $10.4 \%$ - $56.9 \%$ in Asia-pacific areas (Chiang et al., 2016). This shows the resistance property of Pseudomonas against this drug is increasing day by day which may be due to plasmid-mediated transferable enzymes and extended-spectrum enzymes. Massive exploitation of antimicrobials is one of the greatest factors in the selection and persistence of antimicrobial resistance among bacterial pathogens. The limitation of this study was that Pseudomonas was not identified to species level and molecular typing and plasmid profiling were not carried out.

\section{CONCLUSION}

The result shows pus, wound exudates, ear discharges, urine samples exhibit Pseudomonas as etiology of infection. None of the isolates of Pseudomonas spp. were sensitive to cefixime. Pseudomonas isolates reveal the highest sensitivity against piperacillin/tazobactam, amikacin, meropenem, gentamycin. As Pseudomonas spp are gradually becoming resistant to carbapenem and other important antibiotics in many parts of the world, these drugs should be confined to extreme infections and hospital intensive care units to circumvent the speedy emergence of resistant strains. Strict policy on antimicrobial drug overuse, prescription of drugs without susceptibility testing, self-medication practice should be made by the government of Nepal. An effective strategy on the limited and provident use of antipseudomonal agents should be established by hospitals, clinicians, clinical microbiologists, public health officials to convoy empirical therapy.

\section{ACKNOWLEDGEMENTS}

The authors wish to thank Dr. Prson Gautam, Senior Researcher, Institute of Molecular Medicine Finland, University of Helsinki, Finland, for his valuable suggestion to improve the manuscript. The authors also like to acknowledge Kathmandu Model Hospital, Kathmandu, for extending helps in conducting the microbiological analysis for the present study.

\section{CONFLICT OF INTEREST}

The authors declare no conflict of interest.

\section{REFERENCES}

Agbo, E. C., Ejinaka, O. R., Obeta, M. U., Lote-Nwarul, I. E., Ibanga, I. E., \& Godfrey, D. D. (2020). Antimicrobial susceptibility pattern of biofilm forming Pseudomonas aeruginosa isolated from noncritical surfaces in a tertiary healthcare facility in South Eastern Nigeria. American Journal of Public Health Research, 8(3), 87-92.

Aggarwal, R., Chaudhary, U., \& Bala, K. (2008). Detection of extended-spectrum $\beta$-lactamase in Pseudomonas aeruginosa. Indian Journal of Pathology and Microbiology, 51(2), 222-224.

Anil, C., \& Shahid, R. M. (2013). Antimicrobial susceptibility patterns of Pseudomonas aeruginosa clinical isolates at a tertiary care hospital in Kathmandu, Nepal. Asian Journal of Pharmaceutical and Clinical Research, 6(3), 235238.

Chand, Y., Khanal, S., Panta, O. P., Shrestha, D., Khadka, D. K., \& Poudel, P. (2020). Prevalence of some virulence genes and antibiotic susceptibility pattern of Pseudomonas aeruginosa isolated from different clinical specimens. Research Square, pp. 1-12. https://doi.org/10.21203/rs.3.rs-24044/v1

Chiang, T.T., Tang, H. J., Chiu, C. H., Chen, T. L., Ho, M. W, Lee, C. H., Sheng, W. H., \& Yang, Y. S. (2016). Antimicrobial activities of cefoperazonesulbactam in comparison to cefoperazone against 
clinical organisms from medical centers in Taiwan. Journal of Medical Sciences, 36(6), 229-233.

CLSI (2013). Performance Standards for Antimicrobial Susceptibility Testing; Twenty-Fourth Informational Supplement. CLSI document M100-S24. Wayne, PA, USA: Clinical and Laboratory Standards Institute.

Golshani, Z., Mohammad, A., \& Sharifzadeh, A. (2012). Antimicrobial susceptibility pattern of Pseudomonas aeruginosa isolated from patients referring to hospitals. Archives of Hygiene Sciences, 1(2), 48-53.

Javiya, V. A., Ghatak, S. B., Patel, K. R., \& Patel, J. A. (2008). Antibiotic susceptibility patterns of Pseudomonas aeruginosa at a tertiary care hospital in Gujarat, India. Indian journal of pharmacology. 40(5), 230-234.

Kayser, F. H., Bienz, K. A., Eckert, J., \& Zinkernagel, R. M. (Ed.). (2005). Medical microbiology. New York, USA: Thieme, p. 663.

Khan, J. A., Iqbal, Z., Rahman, S. U., Farzana, K., \& Khan A. (2008). Prevalence and resistance pattern of Pseudomonas aeruginosa against various antibiotics. Pakistan Journal of Pharmaceutical Sciences, 21(3), 311-315.

Kireçci, E., \& Kareem, R. D. (2014). Antibiotic susceptibility patterns of Pseudomonas aeruginosa strains isolated from various clinical specimens. Sky Journal of Microbiology Research, 2(2), 13-17.

Kumar, A., Das, S., Anjum, N., Oraon, V., \& Das, S. (2020). Antimicrobial susceptibility pattern of extended spectrum beta-lactamase (ESBL) and nonESBL producing Pseudomonas aeruginosa, isolated from pus samples from a tertiary care hospital in Bihar. International Journal of Current Microbiology and Applied Sciences, 9(6), 36463655.

Maes, P., \& Vanhoof, R. (1992). A 56-month prospective surveillance study on the epidemiology of aminoglycoside resistance in a Belgian general hospital. Scandinavian Journal of Infectious Diseases, 24(4), 495-501.

Mohanasoundaram, K. M. (2011). The antibiotic resistance pattern in the clinical isolates of Pseudomonas aeruginosa in a tertiary care hospital; 2008-2010 (a 3-year study). Journal of Clinical Diagnostic and Research, 5(3), 491-494.
Motbainor, H., Bereded, F., \& Mulu, W. (2020). Multidrug resistance of bloodstream, urinary tract and surgical site nosocomial infections of Acinetobacter baumannii and Pseudomonas aeruginosa among patients hospitalized at Felegehiwot referral hospital, Northwest Ethiopia: a cross-sectional study. BMC Infectious Diseases, 20(1), 92. https://doi.org/10.1186/s12879-020-4811-8

Pathmanathan, S. G., Samat, N. A., \& Mohamed, R. (2009). Antimicrobial susceptibility of clinical isolates of Pseudomonas aeruginosa from a Malaysian hospital. Malaysian Journal of Medical Sciences, 16(2), 28-33.

Ramana, B. V., \& Chaudhury, A. (2012). Antibiotic resistance pattern of Pseudomonas aeruginosa isolated from healthcare-associated infections at a tertiary care hospital. Journal of the Scientific Society, 39(2), 78-80.

Savaş, L., Duran, N., Savaş, N., Önlen, Y., \& Ocak, S. (2005). The prevalence and resistance patterns of Pseudomonas aeruginosa in intensive care units in a university hospital. Turkish Journal of Medical Sciences, 35(5), 317-322.

Sharma, S., Rehan, H. S., Goyal, A., Jha, A. K., Upadhyaya, S., \& Mishra, S. C. (2004). Bacteriological profile in chronic suppurative otitis media in Eastern Nepal. Tropical doctor, 4(2), 102104.

Sivanmaliappan, T. S., \& Sevanan, M. (2011). Antimicrobial susceptibility patterns of Pseudomonas aeruginosa from diabetes patients with foot ulcers. International Journal of Microbiology, 2011, 1-4.

Tripathi, P., Banerjee, G., Saxena, S., Gupta, M. K., \& Ramteke, P. W. (2011). Antibiotic resistance pattern of Pseudomonas aeruginosa isolated from patients of lower respiratory tract infection. African Journal of Microbiology Research, 5(19), 2955-2959.

Ullah, N., Güler, E., Güvenir, M., Arıkan, A., \& Süer, K. (2019). Isolation, identification, and antibiotic susceptibility patterns of Pseudomonas aeruginosa strains from various clinical samples in a University hospital in Northern Cyprus. Cyprus Journal of Medical Science, 4(3), 225-228. 